\title{
Framework on Quality of Service in Mobile Ad Hoc Networks
}

\author{
Siva Krishnaiah. $C^{1}$, Dr Subramanyam. $A^{2}$ \\ ${ }^{1}$ Research Scholar in Rayalaseema University, Kurnool, \\ ${ }^{2}$ Professor \& Dean, Annamacharya Institute of Technology and Sciences, Rajampet.
}

\begin{abstract}
Mobile Ad Hoc Network (MANET) has been continuously self-configuring network composed of a set of mobile devices that can communicate between them without infrastructure connected wirelessly. With the expanding scope of MANET applications the need to support Quality of Service $(Q o S)$ in these networks has been essential. QoS in a network is measured in terms of guaranteed amount of data which a network transfers from one place to another during certain time. To quantitatively measure QoS several related parameters are preferred. This paper studies the issues, challenges, parameters involved in QoS including the classification of QoS approaches and various QoS frameworks are discussed with its scope.
\end{abstract}

Keywords: MANET, Ad hoc Wireless Networks, Quality of Service.

\section{INTRODUCTION}

Mobile Ad Hoc Network (MANET) is a continuously self-configuring network composed of a set of mobile devices which can communicate between them without infrastructure connected wirelessly. Each device in a MANET is free to move independently in any direction, and will therefore change its links to other devices frequently. The primary challenge in building a MANET is equipping each device to continuously maintain the information required to properly route traffic. Such networks may operate by themselves or may be connected to the larger Internet. MANETs are a kind of Wireless Adhoc Network (WANET) that usually has a routable networking environment on top of a Link Layer ad hoc network. Quality of Service is the overall performance of a computer network, particularly the performance seen by the users of the network. QoS in a network is measured in terms of guaranteed amount of data which a network transfers from one place to another during certain time. To quantitatively measure quality of service, several related aspects of the network service are often considered known as parameters of QoS such as error rates, bit rate, throughput, transmission delay, availability, jitter etc., Quality of Service is the ability to provide different priority to different applications, users, or data flows, or to guarantee a certain level of performance to a data flow. Quality of service is particularly important for the transport of traffic with special requirements supporting new applications with even stringent service demands.

II. ISSUES AND CHALLENGES FOR QOS IN MOBILE AD HOC WIRELESS NETWORKS

Providing QoS support in Mobile Ad hoc Networks is an active research area. Some of the characteristics that pose several difficulties in provisioning QoS are dynamically varying network topology, lack of precise state information, lack of a central controller, error-prone shared radio channel, limited resource availability, hidden terminal problem, and insecure medium etc.,[10] How each of these characteristics affects QoS provisioning in ad hoc wireless networks is given below,

2.1 Dynamically varying Network Topology

Since the nodes does not have any restriction on mobility, the network topology changes dynamically. Hence, the admitted QoS sessions may suffer due to frequent path breaks, thereby requiring such sessions to be reestablished over new paths. The delay incurred in reestablishing a QoS session may cause some of the packets belonging to that session to miss their delay targets/deadlines, which is not acceptable for applications that have stringent QoS requirements.

\subsection{Imprecise State Information}

In most cases, the nodes maintain both the link-specific state information and flow-specific state information. The link-specific state information includes bandwidth, delay, delay jitter, loss rate, error rate, stability, cost, and distance values for each link. The flow-specific information includes session ID, source address, destination address, and QoS requirements of the flow such as maximum bandwidth requirement, minimum bandwidth requirement, maximum delay, and maximum delay jitter. The state information is inherently imprecise due to dynamic changes in network topology and channel characteristics. Hence, routing decisions may not be accurate, resulting in some of the real-time packets missing their deadlines.

\subsection{Lack of Central Coordination}

This wireless network does not have central controllers to coordinate the activity of nodes. This further complicates QoS provisioning in ad hoc wireless networks.

2.4 Hidden Terminal problem

The hidden terminal problem is inherent in ad hoc wireless networks. This problem occurs when packets originating from two or more sender nodes, which are not within the direct transmission range of each other, collide at a common receiver node. It necessitates the retransmission of the packets, which may not be acceptable for flows that have stringent QoS requirements. 2.5 Limited Resource Availability

Resources such as bandwidth, battery life, storage space, and processing capability are limited in ad hoc wireless networks. The availability of such resources significantly affects the performance of the QoS provisioning mechanism. Hence, efficient resource management mechanisms are required for optimal utilization of these scarce resources.

\subsection{Insecure Medium}

Due to the broadcast nature of the wireless medium, communication through a wireless channel is highly insecure. They are susceptible to attacks such as 
eavesdropping, spoofing, denial of service, message distortion, and impersonation. Therefore, security is an important issue, without sophisticated security mechanisms, it is very difficult to provide secure communication guarantees.

\section{DESIGN CHOICES FOR QOS SUPPORT}

A design choice is the possibility to achieve the intended objective. The design choice for QoS support gives the required elements to be considered for QoS framework [10]. Some of the possible components for efficient QoS provisioning are

\subsection{Resource Reservation}

QoS resource reservation is responsible for reserving resources at all intermediate nodes along the path from the source to the destination, as requested by the QoS session. QoS resource reservation mechanisms can be broadly classified into two categories: hard state and soft state reservation mechanisms. In Hard state resource reservation scheme, resources are reserved at all intermediate nodes along the path from the source to the destination throughout the duration of the QoS session. If such a path is broken due to network dynamics, these reserved resources have to be explicitly released by a deallocation mechanism. Such a mechanism not only introduces additional control overhead, but may also fail to release resources completely in case a node previously belonging to the session becomes unreachable. The hard state schemes reserve resources explicitly and hence, at high network loads, the call blocking ratio will be high.

Soft state resource reservation mechanisms maintain reservations only for small time intervals. These reservations get refreshed if packets belonging to the same flow are received before the timeout period. The soft state reservation timeout period can be equal to packet interarrival time or a multiple of the packet inter-arrival time. If no data packets are received for the specified time interval, the resources are deallocated in a decentralized manner without incurring any additional control overhead. Thus no explicit teardown is required for a flow and provide high call acceptance at a gracefully degraded fashion.

\subsection{Maintenance of Network Information for Routing}

In the statefull approach, each node maintains either global state information or only local state information. State information includes both the topology information and the flow specific information. If global state information is available, the source node can use a centralized routing algorithm to route packets to the destination. The performance of the routing protocol depends on the accuracy of the global state information maintained at the nodes. Significant control overhead is incurred in gathering and maintaining global state information. On the other hand, if mobile nodes maintain only local state information, distributed routing algorithms can be used. Even though control overhead incurred in maintaining local state information is low, care must be taken to obtain loop-free routes.

In the stateless approach no such information is maintained at the nodes. In this approach neither flow specific nor link specific state information maintained at the nodes. This solves the scalability problem permanently and reduces the burden of storage and computation on nodes, but providing QoS guarantees becomes extremely difficult.

\subsection{QoS provisioning approach (Hard/Soft)}

The QoS provisioning approaches can be broadly classified into two categories: hard QoS and soft QoS approaches. If QoS requirements of a connection are guaranteed to be met for the whole duration of the session, the QoS approach is termed as hard QoS approach. If the QoS requirements are not guaranteed for the entire session, the QoS approach is termed as soft QoS approach. The selection of QoS approach must consider the network dynamics of mobile ad hoc networks.

\subsection{Routing Protocol}

The routing protocol is used to find a path from the source to the destination and to forward the data packet to the next intermediate relay node. QoS routing describes the process of finding suitable paths that satisfy the QoS service requirements of an application. The performance of the routing protocol affects the performance of QoS.

\subsection{Admission Control}

Even though a QoS feasible path may be found, the system needs to ensure whether to actually serve the connection or not. Admission control ensures that there is no perceivable degradation in the QoS being offered to the QoS sessions admitted already.

\subsection{Packet Scheduling}

In the case of multiple QoS connections are active at the same time through a link, the decision on which QoS flow is to be served next is made by the scheduling scheme. The performance of a scheduling scheme is reflected by the percentage of packets that meet their deadlines.

IV. QOS PARAMETERS IN MOBILE AD HOC NETWORKS

QoS parameter is a key element that must be considered in providing Quality of Service solution. The level of QoS and the associated QoS parameters also differ from application to application. The Table.1 gives the list of key QoS parameters and Figure.4 gives list of Metrics to be required for different type of applications. The parameters to be considered for QoS provisioning are [15].

\subsection{Bandwidth}

It is the raw capability of a communications channel to move data through that channel. Typically measured in bits or bytes per second (or some, possibly large, multiple thereof). It refers to how wide the media is, not how fast. 4.2 Throughput

It is the amount of data that actuality moves from one point to another over a given amount of time. Many things effect throughput may include protocol, data loss, latency, and others.

Throughput=amount of data transferred / transmission time

\subsection{Delay or Latency}

It is the time elapsed from the departure of a data packet from the source node to the arrival at the destination node, including queuing delay, switching delay, propagation delay etc.,

Delay $=$ packet arrival time - packet start time 


\subsection{Jitter}

It is a variation in packet delay at the receiver of the information. A network with constant latency has no variation (or jitter).

$$
\sum_{i=1}^{n}\left(\text { Delay }_{i}-\text { Delay }_{i-1}\right) / n-1
$$

\subsection{Packet Loss}

It occurs when one or more packets of data travelling across a computer network fail to reach their destination. Packet loss is typically caused by network congestion. Packet loss is measured as a percentage of packets lost with respect to packets sent.

4.6 Scalability

A system is considered scalable if it is capable of increasing its total output under an increased load when resources (typically hardware) are added.

\subsection{Security}

It is the protection from the unauthorized access and usage of the network resources

\subsection{Availability}

Availability of a system is typically measured as a factor of its reliability. As reliability increases, so does availability. It is the ratio of (A) the total time a functional unit is capable of being used during a given interval to (B) the length of the interval.

\subsection{Energy Conservation}

It considers the reducing of energy consumption through using less energy for a constant service.

\subsection{Error rate}

The number of corrupted bits expressed as a percentage or fraction of the total sent.

\subsection{Packet Delivery Ratio (PDR)}

It is the measure of total number of packets successfully delivered to the destination.

PDR $=$ (No.of packets delivered $/$ No. of packets sent)*100

\begin{tabular}{|l|l|}
\hline \multicolumn{1}{|c|}{ APPLICATIONS } & \multicolumn{1}{c|}{ KEY QOS PARAMETERS } \\
\hline MULTIMEDIA APPLICATIONS & BANDWIDTH, DELAY etC., \\
\hline MILITARY APPLICATIONS & $\begin{array}{l}\text { SECURITY, RELIABILITY, DELAY, BANDWIDTH } \\
\text { etC., }\end{array}$ \\
\hline EMERGENCY/REALTIME APPLICATIONS & AVAILABILITY, RELIABILITY etC., \\
\hline $\begin{array}{l}\text { HYBRID WIRELESS NETWORK } \\
\text { APPLICATIONS }\end{array}$ & $\begin{array}{l}\text { MAXIMUM AVAILABLE LINK LIFE, DELAY, } \\
\text { BANDWIDTH, CHANNEL UTILIZATION etc., }\end{array}$ \\
\hline SENSOR NETWORK APPLICATIONS & BATTERY LIFE, ENERGY CONSERVATION etc., \\
\hline
\end{tabular}

Table 1: Applications and Key QoS Parameters

\section{CLASSIFICATION OF QOS APPROACHES}

QoS classification is made based on the interaction between the routing protocol and QoS provisioning mechanism and the solutions provided based on layers. The classification of QoS approaches [10] are as follows 5.1 Coupled QoS approach

In this Routing protocol and QoS provisioning mechanism closely interact with each other for delivering QoS guarantees[10]. The Figure 1 shows the set of QoS solutions for this category.

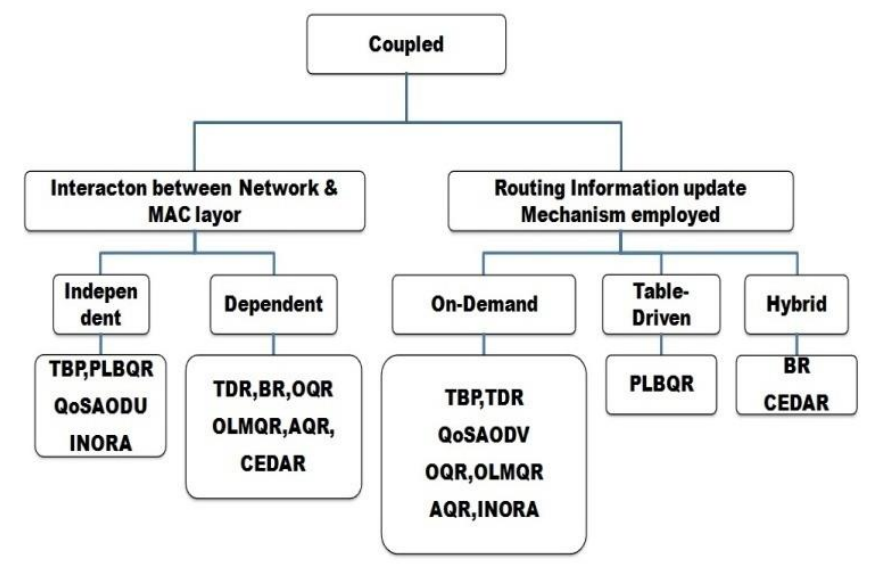

Figure 1: Coupled classification 5.2 Decoupled QoS Approach

In this QoS provisioning mechanism does not depend on any specific routing protocol to ensure QoS guarantees. The Figure 2 shows the set of QoS solutions of this category.

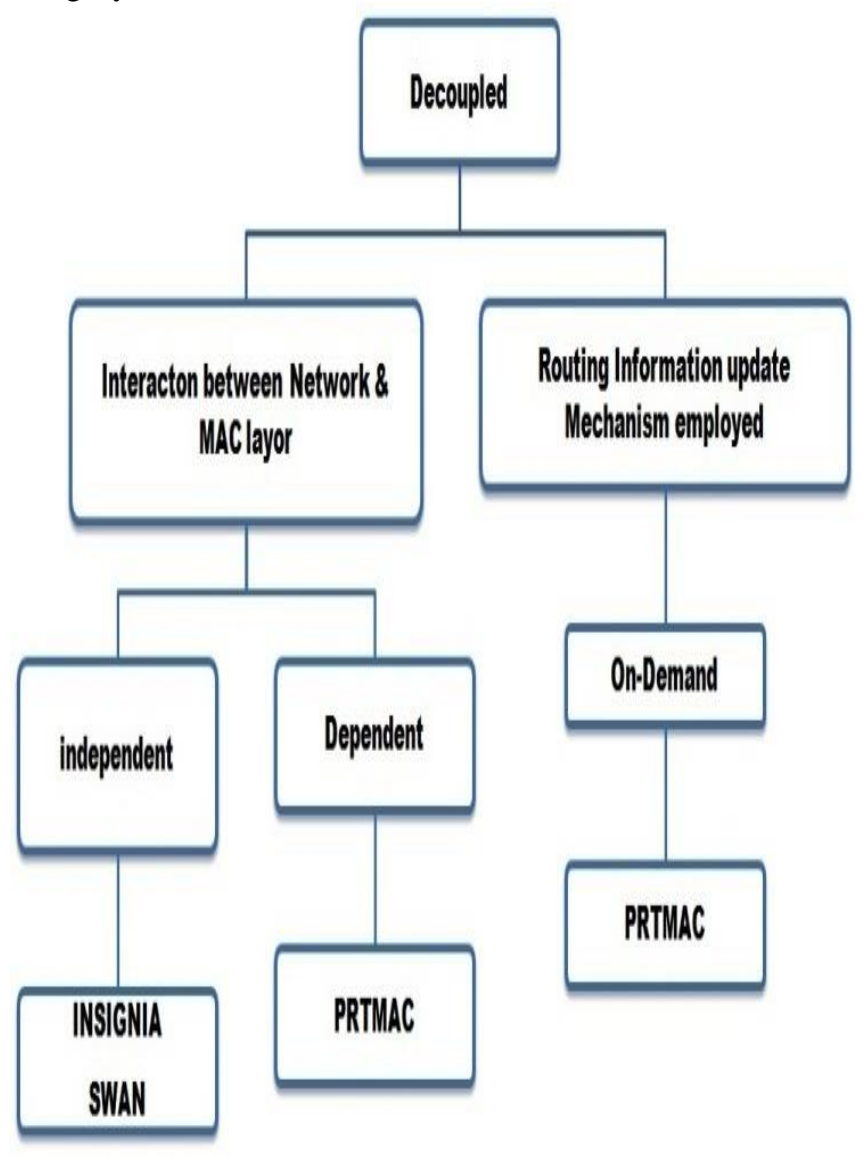

Figure 2: Decoupled Classification

5.3 MAC Layer Perspective QoS Approach

The MAC protocols use channel sensing and random back-off schemes, making them suitable for best-effort data traffic. Figure 3 shows the various solutions of this category.

5.4 Network Layer Perspective QoS Approach

These contain support for ensuring end-to-end resource negotiation, reservation, and reconfiguration.

5.5 Cross-Layer Perspective QoS Approach

These combine the MAC layer and Network layer approaches. 


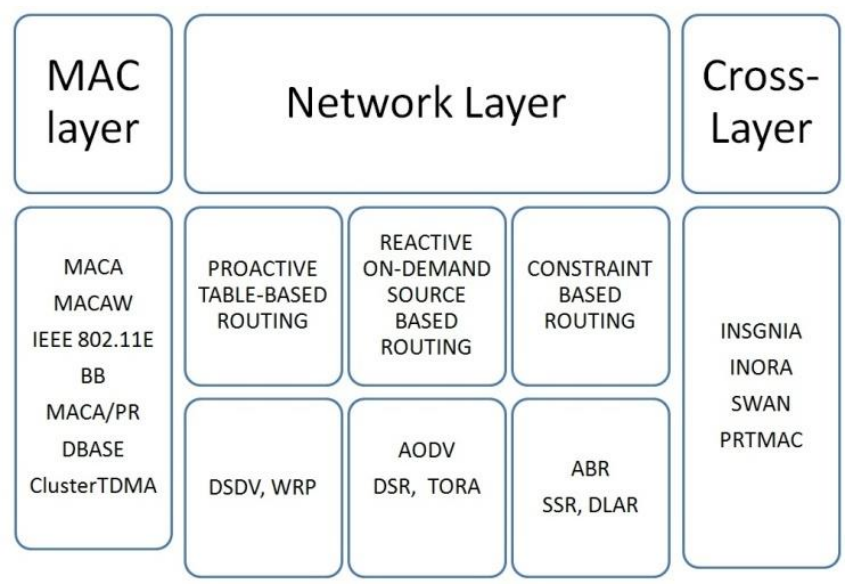

Figure 3: Layered Classification

\section{QUALITY OF SERVICE FRAMEWORKS}

QoS framework is a complete system that attempts to provide the promised services to each user or application. The components in this should cooperate in providing the required services. The key components of QoS framework are

\begin{tabular}{|c|c|}
\hline TIME & \multirow{3}{*}{$\begin{array}{c}\text { AVALABILITY, } \\
\text { RELIABILITY, } \\
\text { STABILITY }\end{array}$} \\
\hline DELAY & \\
\hline JITTER & \\
\hline RESPONSE & PACKET LOSS RATE (PLR) \\
\hline BANDWIDTH & BIT ERROR RATE (BER) \\
\hline SYSTEM LEVEL DATA RATE (SLDR) & \multirow{2}{*}{$\begin{array}{l}\text { MEAN TIME BETWEEN } \\
\text { FAILURES (MTBF) }\end{array}$} \\
\hline BUFFER FULLNESS (BF) & \\
\hline APPLICATON LEVEL DATA RATE (ALDR) & $\begin{array}{l}\text { MEAN TIME TO FAILURE } \\
\text { (MTTF) }\end{array}$ \\
\hline RESDUAL CAPACITY (RC) & \multirow[t]{2}{*}{$\begin{array}{l}\text { MEAN TIME TO REPAIR } \\
\text { (MTTR) }\end{array}$} \\
\hline RAGE CALL ACCEPTANCE RATIO (ACAR & \\
\hline
\end{tabular}

\section{Figure 4: Metrics}

\subsection{QoS Service Model}

It defines the way user requirements are served.

\subsection{QoS Routing}

It is to find all or some feasible paths in the network that can satisfy QoS requirements. The performance of QoS routing affects the performance of QoS framework. It must provide end-to-end QoS guarantees and consume minimal resources, react quickly as network state and flow state changes.

\subsection{QoS Resource Reservation}

It is the resource reservation required by the user or application to achieve desired level of QoS.

\subsection{QoS Access Control}

The Quality of Service access control regarding medium, connection admission and scheduling schemes define network control concepts which are essential in network for better quality of service. QoS admission control ensures that there is no perceivable degradation in the QoS being offered to the QoS sessions admitted already. When multiple QoS connections are active at the same time through a link, the decision on which QoS flow is to be served next is made by the scheduling scheme.
Some Quality of Service frameworks are,

i. QoS Hierarchical Queuing Framework (HQF) of Cisco HQF [7] feature enables you to manage Quality of Service at three different levels: the physical interface level, the logical interface level, and the class level for QoS queuing and shaping mechanisms by using the Modular QoS Command-line (MQC) interface to provide a granular and flexible overall QoS architecture.

ii. On-Demand QoS Path framework (ODP)

This provides end-to-end QoS guarantees to individual flows with minimal overhead, while keeping the scalability characteristic of DiffServ. ODP [12] exercises per-flow admission control and end-to end bandwidth reservation at the edge of the network and only differentiates traffic classes in the core of the network. In addition, to adapt to dynamically changing traffic load, ODP monitors the bandwidth utilization of the network and performs dynamic bandwidth reconfiguration in the network core.

iii. IntServ

IntServ framework implemented the RSVP (Resource Reservation Protocol) to reserve bandwidth at routers along the path of a flow [14]. When a flow arrives with a QoS requirement, the ingress edge router initiates the path establishment process by sending a PATH message to the destined egress edge router. The egress edge router responds by sending a RESV message back to the ingress router and tries to reserve the bandwidth required to meet the requested QoS along the path to the source ingress edge router. Core routers on the path configure their traffic control mechanisms such that each admitted flow is guaranteed to receive the bandwidth reserved, and thus the requested QoS. Through this per-flow based hop-by-hop signaling, IntServ provides end-to-end QoS guarantees. It is best applicable for small sized adhoc wireless networks. iv. DiffServ

In the DiffServ framework flows are grouped into a small number of classes at the boundary of a network and the routers within the network merely implement a suite of scheduling/buffering mechanisms based on the classes. This per class approach simplifies a router's functionalities and reduces the states that a router has to keep. The per-class approach also removes the per-flow QoS signaling overhead. Thus, DiffServ is more scalable than IntServ. However if bandwidth provisioned on a path is used only by a single flow, this scheme loses its advantages and degrades to IntServ. It offers scalability by aggregating flows into a limited number of service classes.

v. DiffServ with Endpoint Admission Control (EAC)

In this framework an admission control function is provided over DiffServ network by means of the Endpoint Admission Control. EAC builds upon the idea that admission control can be implemented purely in an endto-end manner, involving only the source and destination hosts. At connection setup, each source-destination pair starts a probing phase to determine whether a connection can be admitted to the network. The source node sends probing packets that reproduce the traffic characteristics of the connection to be established. Upon reception of the first probing packet, the destination host starts monitoring probing packets' statistics (e.g., loss ratio, inter-arrival 
times) for a given period of time. At the end of the measurement period, the destination makes the decision as to whether to admit or reject the connection and notifies the decision to the source. Although this scheme is scalable since it does not involve inner routers, there are a number of downsides. Measurements taken in a short probing time may not capture stationary network states, and thus, the admission control decision is made based on a snapshot of the network that may not reflect the true status of network congestion. On the other hand, if measurements are taken in a longer probing period, the admission control process will be very slow. In addition, since probing packets are actually transmitted through a network during the measurement period, the probing packets increase the traffic load and contribute to network congestion.

vi. DiffServ-aware MPLS Traffic Engineering (DS_TE)

In DiffServ aware MPLS Traffic Engineering (DS_TE) DiffServ is complemented by MPLS Traffic Engineering mechanisms that operate on an aggregate basis across all DiffServ traffic classes. DS_TE further aggregate traffic classes to class types (CTs) and provision bandwidth for each CT in the core of a network. In order to reduce flooding overhead of link state advertisements, Inter Gateway Routing Protocol (IGP) extension of per classtype Link State Advertisements (LSA) is used to exchange information on the available bandwidth for each class type. When an edge router makes an admission control decision, the edge router chooses a path for the incoming flow using Constraint Based Routing. Although the scalability of IGP LSAs is improved by propagating information on a per-class-type basis instead of on a perclass basis, no bandwidth provisioning is enforced for each traffic class within a class type. Flows of different traffic classes within a class type may interfere with each other.

\section{vii. AQuaFWiN (Adaptive QoS Framework for \\ Multimedia in Wireless Networks)}

This framework proposed by Bobby Vandalore, Raj Jain, Sonia Fahmy and Sudhir Dixit[8]. This framework is hierarchical in nature with cluster of mobile end hosts connected to a base station, which are connected to a supervisory node, which in turn is connected to the wired infrastructure. The changing conditions in wireless due to interference and possibly mobility, entitle that the realtime applications needing stringent QoS should be adaptable. The framework uses a generic feedback mechanism to support adaptability at all layers of the wireless network.

viii. WAMIS (Wireless Adaptive Multimedia Information System)

The adaptive mobile multimedia networks architecture for a "3M" environment (real-time Multimedia, Multi- hop and Mobile) was designed and implemented in the WAMIS (wireless adaptive multimedia information system) project at UCLA. Most of the other architectures include only two of the M's. The WAMIS project used an integrated design, evaluation and prototyping approach. WAMIS uses a wavelet-transform based adaptive video compression algorithm.

ix. INORA
It is a framework that makes use of INSIGNIA in band signaling and TORA routing protocol [1]. The QoS resource reservation mechanism, which is a soft state reservation mechanism, interacts with the routing protocol to deliver QoS requirements. INORA can search multiple paths with lesser QoS guarantees. In this no resources are reserved before the actual data transmission begins and packets have to be transmitted as best effort packets in case of admission control failure at the intermediate nodes.

x. SWAN (Stateless Wireless Adhoc Network)

This framework uses a local rate control mechanism for regulating injection of best-effort traffic into the network, a source-based admission control while accepting new real-time sessions, and an Explicit Congestion Notification (ECN) mechanism for dynamically regulating admitted real-time sessions. Changes $\mathrm{n}$ topology and network conditions, even node and link failures, does not affect the operation of the SWAN control system. It uses feedback based control mechanisms to regulate real-time traffic at the time of congestion in the network. This cannot provide hard QoS guarantees due to lack of resource reservation at the intermediate nodes. An admitted real-time flow may encounter periodic violations in its bandwidth requirements.

\section{xi. PRTMAC (Proactive Real-Time MAC)}

It is a cross-layer framework, with an on-demand QoS extension of DSR routing protocol at the network layer and real-time[2] MAC protocol at the MAC layer. It is a tightly coupled solution which requires the bandwidth reservation and bandwidth availability estimation services from underlying MAC protocol. PRTMAC provide better real-time traffic support and service differentiation in high mobility adhoc wireless networks. But it is not a better solution for lower-power and resource-constrained handheld devices, having another channel.

xii. DS-SWAN (Differentiated Services-Stateless Wireless Ad hoc Networks)

This framework is a model developed for E2E QoS provisioning in wireless ad hoc networks connected to fixed infrastructure. It is a combination of SWAN and DiffServ models, for wireless and wired networks, respectively parameters in swan model are dynamically adjusted to conditions in wireless and wired network. If the delay is larger than some predefined value the model aggressively shaping of best effort traffic.

xiii. 2LQoS (Two-Layered Quality of Service)

This framework is actually a QoS routing algorithm with the differentiation and shaping of ingress traffic. Path discovery is done based on parameters regarding power consumption and mobility, while selection of the oath is done based on delay and bandwidth. This model supposes three service classes.

xiv. CLQM (Cross-Layer QoS Mapping)

This model provides service differentiation in MANET environment where QoS parameters are mapped, to four proposed service classes, between three layers of protocol stack.

xv. QoSMMANET (QoS Management in Mobile Ad hoc Networks)

This framework represents a QoS support for real-time traffic in highly mobile and ad hoc environments. The 
proposed framework consists of the three building entities regarding routing, traffic differentiation and bandwidth allocation.

xvi. MMWN (multimedia support for mobile wireless networks)

In this Servers are used to provide the various functionalities such as location management and QoSaware route generation[9]. A "location manager" is present in each cluster, which maintains location information of endpoints of that cluster. This also assists in locating endpoints within and outside of the cluster. A "QoS-manager" in each cluster computes the QoS attributes to be advertised in the link-state information xvii. INSIGNIA

This framework is an IP-based quality of service framework that supports adaptive services in Mobile Adhoc Networks[5]. The framework is based on an inband signaling and soft-state resource management approach that is well suited to supporting mobility and end-to-end quality of service in highly dynamic environments where the network topology, node connectivity, and end-to-end quality of service are time varying. Architecturally INSIGNIA is designed to support fast reservation, restoration, and end-to-end adaptation based on the inherent flexibility and robustness and scalability found in IP networks. This framework provides an integrated approach to QoS by combining in-band signaling, call admission control and packet scheduling. It supports only adaptive applications. It is not best suitable for real-time application that has stringent QoS requirements.

xviii. CEQMM (Complete and Efficient Quality of Service Model for MANET)

It is a hybrid scheme, combining per-flow (for traffic with highest priority) and per-class QoS provisioning (traffic with other priorities).Model consists of priority classifier, active queue management, packet scheduler and congestion avoidance mechanisms [4].

xix. iMAQ (Integrated Mobile Ad hoc QoS)

This framework is cross-layer architecture for transport of multimedia data in MANET. Middleware communicates with network layer and applications for higher QoS level of the whole system middleware uses information of nodes location provided by the network layer to predict partitioning of the network. After predicted portioning, data is replicated and diverted depending on the location and mutual arrangement of the nodes.

xx. FQMM (Flexible QoS Model for Mobile Adhoc Networks)

It is a hybrid service model and based on IntServ and DiffServ model[3]. This model classifies nodes into ingress node (source), interior node (intermediate relay node) and ingress node (destination) on a per flow basis. This model is based on assumption that the percentage of flows requiring per flow QoS is much less than that of low-priority flows which can be aggregated into QoS classes. FQMM combines the reservation procedure for high priority traffic with service differentiation for lowpriority traffic. Thus, FQMM provides the ideal QoS for per flow and overcomes the scalability problem by classifying the low-priority traffic into service classes. This addresses the scalability problem, but it cannot solve other problems such as, decision upon traffic classification, allotment of per flow or aggregated service for the given flow, amount of traffic belonging to per flow service, and scheduling or forwarding of the traffic by the intermediate nodes.

\section{CONCLUSION}

This paper focus on various QoS issues \& challenges, parameters and frameworks present for QoS enhancement. Most of the frameworks concentrate on per-flow basis and/or per class basis for scalability issues. Use of location, mobility, and power consumption, probability of resources, bandwidth, and route availability are some major issues which are need to be considered by the researchers. Supporting for multiple levels of services in QoS routing, security and availability are some other challenges in QoS routing. Efficient and effective solutions to these issues will facilitate the design and development of QoS support in MANET's.

\section{REFERENCES}

[1] D.Dharmaraju, A.R.Chowdhury, P.Hovaresti, and J.S.Baras, "INORA-A Unified signaling and Routing Mechanism for QoS Support in Mobile Ad Hoc Networks," Proceedings of ICPPW 2002, pp.86-93, August 2002.

[2] T.Sandeep, V.Vivek,B.S.Manoj, and C.Siva Ram Murthy , "PRTMAC: An Enhanced Real-Time Support Mechansm for Tactical Ad Hoc Wireless Networks," Technical Report, Department of C.S.E, IIT,Madras,June,2001.

[3] H.Xiao,K.G.Seah,A.Lo, and K.C.Chua, "A Flexible Quality of Service Model for Mobile Ad Hoc Networks," Proceedings of IEEE Vehicular technology Conference, vol,1, pp.445-449, May 2000.

[4] H.Badis, K.Al Agha, "CEQMM: a complete and efficient quality of service model for MANETs", Proceedings of the $3^{\text {rd }}$ ACM international workshop on Performance evaluation of wireless ad hoc, sensor and ubiquitous networks-PE-WASUN'06,Malaga, Spain,2006,pp.25-32

[5] S.B. Lee, A.Gahng-Seop, X.Zhang, and A.T. Campbell, "INSIGNIA: An IP-Based Quality of Service Framework for Mobile Ad Hoc Networks," Journal of Parallel and Distributed Computing, vol.60, No.4, pp.374-406, April 2000.

[6] W.K.G.Seah,H.X.Tan,"Quality of Service in Mobile Ad Hoc Networks," in Encyclopedia of Internet Technologies and Applications, M.Freire and M.Pereira , IGI-Gloabal, 2008, pp.441-448.

[7] QoS: Hierarchical Queueing Framework Configuration Guide, Cisco,IOS Release 15M\&T, Published: January 28, 2013

[8] Bobby Vandalore, Raj Jain, Sonia Fahmy, Sudhir Dixit “ AQuaFWiN: Adaptive QoS Framework for Multimedia in Wireless Networksand its Comparison with other QoS Frameworks,"

[9] R. Ramanathan and M. Steenstrup. Hierarchically organized, multihop mobile wireless networks for qualityof-service support. Mobile Networks and Applications,3(1):110-119, 1996.

[10] C.Siva Ram Murthy,B.S.Manoj "Ad Hoc Wireless Networks architectures and protocols," book, pearson educaton .

[11] A. Alwan, R. Bagrodia, N. Bambos, M. Gerla, L. Kleinrock,J. Short, and J. Villasenor." Adaptive Mobile Multimedia Networks." IEEE Personal Communications, 3(2):35-51, April 1996 
[12] Mei Yanga, Yan Huanga, Jaime Kimb, Meejeong Leec, Tatsuya Sudaa, Matsubara Daisuked "An End-to-End QoS Framework with On-Demand Bandwidth Reconfiguration,” IEEE INFOCOM 2004.

[13] Mrs. Manisha P. Navale, Prof. G.T. Chavan, "Survey on QoS improving method in MANET,", IJERT, Vol.3 Issue 12, Dec,2014.

[14] Lie Chen and Wendi B. Heinzelman, "A Survey of Routing Protocols that Support QoS in Mobile Ad Hoc Networks," IEEE Network, Nov-Dec, 2007

[15] Prasant Mohapatra, Jian Li and Chao Gui,"QoS in Mobile Ad Hoc Networks," IEEE Wireless Communications, June-2003. 